\title{
FALSE MEMORIES AND IMPRESSIONS OF PERSONALITY
}

\author{
Leonel Garcia-Marques, Mário B. Ferreira, and Ludmila D. Nunes \\ University of Lisbon \\ Margarida V. Garrido \\ ISCTE Lisbon University Institute \\ Teresa Garcia-Marques \\ ISPA, Lisbon
}

\begin{abstract}
We extended the false memories paradigm to the study of impressions formation. Traits most commonly used in describing person-targets were employed to identify the four clusters underlying the implicit theory of personality semantic structure (intellectual positive and negative; social positive and negative). Finally, we developed lists including semantic neighbors of the traits closest to the clusters' centroid and athematic (non-trait) words. Participants were presented with these lists and instructed to either form an impression of a person described by those words or simply to memorize them. Impression formation relative to memory participants produced higher levels of false memories of lures corresponding to the same cluster of the list traits and the reverse pattern was found for a-thematic words. Parallel results from a gist test suggest that forming impressions implies the activation of a specialized associative memory structure underlying the referred bi-dimensional implicit theory of personality (Rosenberg, Nelson, \& Vivekananthan, 1968).
\end{abstract}

"Key words that is [sic] my life: Energy. Strength. Friendship. Socialising. Family. Goals. Art. Design. Love. Mountains. Air. Intimacy. Productiveness. Smile. Laughter and Dancing."

-Personal profile featured on a matchmaking website

\footnotetext{
We want to thank Dr. Henry L. Roediger, III for his useful commentaries and suggestions. Preparation of this article was supported in part by the Grant PTDC/PSI/66864/2006 from the FCT (Portugal).

Correspondence concerning this article should be addressed to Dr. Leonel Garcia-Marques, Faculdade de Psicologia e de Ciências da Educação, Universidade de Lisboa, Alameda da Universidade, 1649-013 Lisboa, Portugal. E-mail: garcia_marques@sapo.pt.
} 
Our impressions of others serve us well in social interaction. They tip us about who to trust, whom to vote for, if person $\mathrm{X}$ is right for the job, whether that used cars salesman is presenting us with a lemon or a good deal. The idea that these impressions are grounded on general templates or semantic structures has a relatively long bearing in the literature (e.g., Asch, 1946). However, the specific characterization of the cognitive nature of these structures has been only partially attempted (Cantor \& Mischel, 1977, 1979; Hamilton, Katz, \& Leirer, 1980; Schneider \& Blankmeyer, 1983). In this article, we advance the hypothesis that these structures are specialized associative memory structures that share main features with other such structures, endowed with the same flexible powers and prone to display corresponding performance costs.

One paradigm that has recently been successfully used to explore these associative structures is the DRM (Deese-Roediger-McDermott; Deese, 1959; Roediger \& McDermott, 1995). Our goals are to adapt this paradigm to study the Implicit Theories of Personality, to explore its associative nature and to suggest a new outlook to both impression formation and associative memory structures.

\section{FALSE MEMORIES IN THE DEESE-ROEDIGER-MCDERMOTT (DREAM) PARADIGM}

Roediger and McDermott (1995) replicated and extended a paradigm first introduced by Deese (1959), the so-called DRM or DREAM (an acronym for Deese-Roediger-McDermott). In this paradigm, the words that are most often free associated with a critical concept are used to form a stimulus list (e.g., the words, sour, candy, sugar, bitter, good, taste, tooth, nice, honey, soda, chocolate, heart, cake, tart and pie formed the sweet list). When participants hear lists such as this and later are asked to recall them, they very often falsely recollect the (nonpresented) critical word (sweet). The level of false recall of the critical word is equivalent to the level of veridical recall of words presented in the middle of the list. In recognition tests, the level of false recognition of the critical word is even greater and is accompanied by strong phenomenological and source illusions (Payne, Ellie, Blackwell, \& Neuschatz, 1996). Dual-process theories of false memories like the Activation-Monitoring framework explain these results as the outcome of two opposing processes, a spreading-activation process that describes how activation converges from the associates to the critical concept and the failure of a deliberate monitoring process that is supposed to discriminate between presented from non-presented information (Roediger, Watson, McDermott, \& Gallo, 2001).

Thus, false memories are no longer taken to be bizarre or sui generis effects but, instead represent the cost of flexible associative memory structures that possess considerable learning powers and inference skills (Roediger, 1996). Subsequent research using the DRM paradigm has supported this perspective. In fact, false memories have been obtained with not only semantic associates of a critical concept (Deese, 1959; Roediger \& McDermott, 1995), but also with phonological neighbors (Sommers \& Lewis, 1999) or even with table-related numerical near-neighbors (Pesta, Sanders, \& Murphy, 2001). 


\section{THE IMPLICIT THEORY OF PERSONALITY AS AN ASSOCIATIVE MEMORY STRUCTURE}

From the very beginning of the field, researchers concerned with the way we form impressions of personality have been keenly aware that laypeople and expert judges alike go beyond the information given (Thorndike, 1920). In fact, research has shown that perceivers have strong expectations about what personality traits "go together" (Bruner, Shapiro, \& Tagiuri, 1958). These expectations were dubbed as implicit theory of personality (Bruner \& Tagiuri, 1954). Moreover in perceivers' judgments, the correlations between such traits that "go together" tend to be overestimated (Berman \& Kenny, 1976) and they are as pervasive when perceivers judge total strangers as when they judge familiar targets (Passini \& Norman, 1966). Subsequent research relying on data reduction techniques such as multidimensional scaling (Rosenberg et al., 1968) and cluster analysis (Cantor \& Mischel, 1977; Rosenberg \& Sedlak, 1972) obtained generally convergent results in defining the semantic structure underlying impressions of personality. Such structure can be conceived as a bi-dimensional semantic space formed by two largely independent evaluative dimensions: one intellectual and one social—resulting in four clearly differentiable clusters: a social positive, a social negative, an intellectual positive, and an intellectual negative cluster.

In addition, further research has shown that the recruitment of the semantic structure underlying impressions of personality is conditional to impression formation and that this process carries reliable mnemonic consequences. Namely, Hamilton and colleagues (1980) have shown that when forming impressions about a target described in several sentences illustrating different traits, participants (relative to controls instructed to simply memorize the same information) perform better at recall and exhibit higher clustering of the behaviors that illustrate the same traits.

More recent research has corroborated the critical importance of these two dimensions for person impressions (for a review, see Fiske, Cuddy, \& Glick, 2007). From this perspective, impression formation can be conceived as a process of placing a target in this semantic space (Brown, 1986). This conception of the process underlying personality impressions can explain why participants often go beyond the information given during impression formation, actively inferring non-presented traits and incorporating them into their impressions of personality.

\section{FALSE MEMORIES AND IMPRESSION FORMATION}

As aforementioned, false memory effects have been shown in a variety of associative structures. We hypothesized that these effects could also originate from impression formation. As Brown (1986) suggested when people form impressions of a target's personality from a series of stimulus traits, they attempt to position the target in the intellectual/social semantic space described by 
Rosenberg and colleagues (1968). We hypothesize that impression formation encoding processes involve the assemblage of a specific semantic trait space before placement of the target in that space. For instance, if a person is presented with a number of positive intellectual traits, the subsequent activation of these traits would gradually converge on their non-presented semantic neighbors that loaded more heavily in the intellectual dimension and possess positive valence. In order to test this hypothesis, we composed four study lists that mainly correspond to the four clusters from the bi-dimensional implicit theory of personality (Rosenberg et al., 1968) and presented them to our participants, such that each participant was only presented with one of them. At test, we assessed recognition memory for critical non-presented traits that best represent these four clusters. We expect false alarms to be higher for traits that belong to the same cluster of those included in the list than for traits that belong to other clusters. However, because the recruitment of the semantic structure or space underlying impressions of personality is hypothesized to be conditional on impression formation, we expected this difference to be much greater for impression formation relatively to memory participants.

Before we describe the present experiment more fully, a caveat is in order. Since the study list itself was composed by highly diagnostic trait words, it could inadvertently induce impression formation goals (for a similar case, see Lichtenstein \& Srull, 1987; for an example of the implicit induction of impression formation goals, see Chartrand \& Bargh, 1996). In fact, as indicated by pilot testing, participants in the memory condition would often engage in spontaneous impression formation. To prevent such possibility, we also included a-thematic words (not related to personality traits) in the study lists and associates of these a-thematic words in the recognition test. To make these a-thematic words plausible descriptors of personality, impression formation participants were told that the study list was composed of words provided by people who knew the target well.

Thus, we predicted a higher rate of false memories under impression formation relative to memory conditions because the recruitment of the semantic structure underlying impressions of personality is conditional on impression formation. However, it can be argued that fewer false memories under memory instructions are merely due to enhanced source monitoring at retrieval. Memory participants might simply be better at discriminating presented from non-presented items than impression formation participants, because they encoded more distinctive features of the list items at study. They then could use their failure to remember distinctive features at retrieval to decide that they had not seen the item. To disentangle these potential different explanations we added a "gist" recognition test in which participants are asked to mark all items presented in the study phase plus all items that "could have been presented" because they are somehow related to presented items (Brainerd, Wright, Reyna, \& Payne, 2002). Such a test makes remembering distinctive item features irrelevant, and thus prevents this "distinctiveness heuristic" at retrieval from contributing to performance. As standard and gist recognition tests differ dramatically in their monitoring requirements, the comparison between the results of these two tests has been used to differentiate between 
alternative accounts of the conditions in which false memories decrease (Hege \& Dodson, 2004; Schacter, Cendan, Dodson, \& Clifford, 2001). In our case, the proposal of a specialized semantic structure that is assembled under impression formation goals will be empirically supported if the results of both tests are identical (i.e., greater false alarms in the impression formation than in the memory group). Similar performance in the two conditions will suggest a specific relational deficit for memory participants because even when they are tested under conditions that minimize monitoring requirements, fewer false alarms (highly diagnostic for impression formation) would occur. On the other hand, if the potential difference between impression formation and memory found under standard recognition test conditions disappears in the gist test, this will suggest that the difference is merely a function of a higher efficacy of monitoring of memory relative to impression formation participants. That is, encoding the items for a subsequent memory test facilitates later monitoring and rejection of non-presented critical items.

\section{METHOD}

\section{PARTICIPANTS AND DESIGN}

One hundred and forty-nine (123 female, 26) students from the University of Lisbon were assigned to a 2 types of encoding context (memory vs. impression formation) X 4 lists (social-positive vs. social-negative vs. intellectual-positive vs. intellectual-negative) $X 2$ types of items (personality traits vs. a-thematic words) mixed design, the last factor being within-participants. The number of participants assigned to each of the eight between-participants conditions varied between 17 and 21. Participants received course credits in exchange for their collaboration.

\section{STIMULUS MATERIAL}

A first sample of 25 students was first asked to provide short trait-based descriptions of liked and disliked persons (both personal acquaintances and persons they did not know personally). From these descriptions we chose the most frequent 40 positive and 40 negative traits. Following Rosenberg et al. (1968), a different sample of 27 students sorted the 80 traits in 12 sets according to their likelihood of co-occurrence in the same person. A multidimensional analysis basically reproduced the bi-dimensional structure identified by Rosenberg et al. (1968) (stress = .21). A 4-Way Cluster Analysis identified the four expected clusters that result from the combination of the two evaluative dimensions: social-positive (e.g., friendly, generous, kind), social-negative (e.g., conceited, selfish, aggressive), intellectual-positive (e.g., cultured, smart, determined), and intellectual-negative (e.g., incompetent, lazy, irresponsible). We used this 4-Way Cluster Analysis to select the 15 words of each cluster closest to its centroid. The 5 words closest to each centroid were used as the critical 
lures (never to be presented to participants) and the 10 next words formed the four lists (i.e., intellectual-positive, intellectual-negative, social-positive, and social-negative). We also selected six a-thematic words ${ }^{1}$ not related to personality traits and included them in each of the four lists we used, obtaining four lists of 16 words each (ten personality traits and six a-thematic words). The a-thematic words were common to every presented list.

The recognition test contained 43 items: the 20 critical lures (5 per cluster/ list); 5 impression-irrelevant lures; 10 non-presented a-thematic words (including 6 semantic associates of the presented a-thematic words); 3 presented a-thematic words; and 5 presented traits. So, the 5 presented traits included in the recognition test varied according to the studied list, but the rest of the test remained equal to every participant.

The gist test was identical to the standard recognition test except for sequence of presentation which was separately randomized for each test.

\section{PROCEDURE}

The experiment was run in small groups of 4 to 10 participants. In the impression formation condition, participants were asked to form an impression of personality of a target person described by a set of words provided by people who were well acquainted with the target. The participants were alerted to the fact that those words could be adjectives or a-thematic nouns because these were words that "people who know the target well associate with him." An example of how lists were formed was provided to participants. Namely, participants read about a man "who works in an office and often forgets his pen and insistently borrows his colleagues'. When asked to provide words that come to mind when thinking about him, his close friends and colleagues provided associations such as office, pen, persistent, and forgetful."

After the participants had read these instructions, the experimenter announced the name of the target and they heard the CD-recorded list of the 16 words. Subsequently participants were instructed to mentally revise their impressions and performed this task for 90 seconds. In the memory condition, participants were presented with a list of 16 words and they were instructed to memorize them. After presentation of the list, participants rehearsed the presented items for 90 seconds as a preparation for the upcoming memory test. After this learning phase, the procedure was identical in both conditions. Participants performed a distracter task for 10 minutes, followed by the standard recognition test and then the gist test. The instructions for the standard recognition test read:

"You will be presented with a number of words. Some of these words were included in the audio recording you previously listened to, others are new. Please read each word as it appears on the screen and indicate whether that was included in the audio recording or not. If you recognize the word

1. The selected a-thematic words were: clock; phone; count; blue; wardrobe; and rose. These words were selected to avoid previous associations to personality traits or mood (at least, in Portuguese). 
press the green key (indicating that you consider that the word was included in the audio recording you listened to), but if you think the word is new, press the red key (indicating that you consider that the word was not included in the audio recording). Please press the green key only if are reasonably certain that the word was included in the audio recording."

The instructions for the gist recognition test read:

"You will be presented with a number of words. Some of these words were included in the audio recording you previously listened to, others are new. When you recognize a word that was presented before or you think that the word could be considered an example of a theme or concept included in the audio recording you listened to previously (even if the word itself was not included in the audio recording) press the green key. If you consider the word to be new because it was not included in the audio recording and does not exemplify any theme or concept included in the recording, please press the red key."

At the end of the sessions, participants were fully debriefed and thanked.

\section{RESULTS AND DISCUSSION}

Participants performed very well in the recognition test as the level of hits always greatly exceeded the level of false memories (see Table 1). We first analyzed hits computing a mixed-model Processing Goal (Impression Formation vs. Memory) X 4 Lists (Social Positive vs. Social Negative vs. Intellectual Positive vs. Intellectual Negative) X 2 Type of Hits (Traits vs. A-thematic words) ANOVA, the last factor being within-participants and found only a main effect for Type of Hits, $F(1,141)=13.93, p=.0001, M S e=.04, \eta^{2}=.09$, showing that participants correctly identified more presented traits than a-thematic words $(M=.86$ vs. $M=.76)$. Our critical prediction regarded an increased level of false memories that semantically correspond to the cluster of the presented list under impression formation when compared to memory settings (see Table 1 ). To test this prediction we recoded the obtained false memories into list matching (proportions of false memories that corresponded to the same trait cluster of the items of the presented list), list mismatching (proportions of false memories that corresponded to a different trait cluster), and a-thematic associates (non-presented items that were associated with the a-thematic presented words). We then performed a mixed-model 2 Processing Goal (Impression Formation vs. Memory) X 4 Lists (Social Positive vs. Social Negative vs. Intellectual Positive vs. Intellectual Negative) X 3 Type of False Memories (List Matching vs. List Mismatching vs. A-thematic Associates) ANOVA, the last factor being within-participants. We obtained a significant Type of False Memories main effect. This Type of False Memories effect, $F(2,282)=222.02, p$ $=.0001, \mathrm{MSe}=.02, \eta^{2}=.58$, revealed that, as expected, List Matching $(M=.34)$ were much more likely than List Mismatching $(M=.05)$ or A-thematic Associates $(M=.03)$ False Memories. More interestingly, we also obtained the critical 
TABLE 1. Proportions of Different Types of False Memories by List

\begin{tabular}{|c|c|c|c|c|}
\hline \multirow[b]{2}{*}{ List Items (Hits) } & \multicolumn{4}{|c|}{ Lists } \\
\hline & Social Positive & Social Negative & $\begin{array}{r}\text { Intellectual } \\
\text { Positive }\end{array}$ & $\begin{array}{r}\text { Intellectual } \\
\text { Negative }\end{array}$ \\
\hline \multicolumn{5}{|l|}{ Impression Formation } \\
\hline Trait Items & .95 & .83 & .82 & .84 \\
\hline A-thematic Items & .75 & .74 & .82 & .81 \\
\hline \multicolumn{5}{|l|}{ False Memories } \\
\hline Social Positive & .40 & .00 & .07 & .04 \\
\hline Social Negative & .00 & .34 & .00 & .04 \\
\hline Intellectual Positive & .22 & .04 & .46 & .01 \\
\hline Intellectual Negative & .00 & .05 & .00 & .40 \\
\hline A-thematic Associates & .00 & .04 & .00 & .02 \\
\hline \multicolumn{5}{|l|}{ Memory } \\
\hline Trait Items & .90 & .84 & .80 & .81 \\
\hline A-thematic Items & .75 & .77 & .77 & .71 \\
\hline \multicolumn{5}{|l|}{ False Memories } \\
\hline Social Positive & .21 & .02 & .05 & .01 \\
\hline Social Negative & .00 & .30 & .01 & .06 \\
\hline Intellectual Positive & .23 & .03 & .31 & .06 \\
\hline Intellectual Negative & .00 & .17 & .01 & .32 \\
\hline A-thematic Associates & .06 & .06 & .01 & .04 \\
\hline
\end{tabular}

Note. Proportions of false memories that corresponded to the same cluster of the Implicit Theory of Personality of the items of the presented list are in bold. $N=149$

Processing Goal X Type of False Memories interaction, $F(2,282)=10.92, p=$ $.0001, M S e=.02, \eta^{2}=.03$. This interaction highlighted the fact that whereas the level of false memories was much higher for Impression Formation relative to Memory participants in the case of List Matching $(M=.40$ vs. $M=.28), t(141)$ $=2.69, p=.0160$, this difference disappeared for List Mismatching $(M=.04$ vs. $M=.05, \mathrm{p}<.25)$ and reversed for A-thematic Associated false memories $(M=$ .02 vs. $M=.04, t(141)=2.78, p=.0031)$. Thus, as predicted, impression formation participants were much more prone to accept false items from the Implicit Theory of Personality trait space that corresponded to the presented trait list. This result corroborates our argument that impression formation involves the activation of a specific semantic structure hypothesized in the implicit theory of personality followed by the placement of a target on this trait space. Moreover, the fact that this difference reversed for non-presented associates of the presented a-thematic words speaks to the specificity of the process underlying impression formation. We will return to this reversal later.

As aforementioned, participants also completed a gist recognition test after the standard recognition test. The resulting data are presented in Table 2. We computed a mixed-model 2 Processing Goal (Impression Formation vs. Memory) X 4 Lists (Social Positive vs. Social Negative vs. Intellectual Positive vs. Intellectual Negative) X 2 Type of Hits (Traits vs. A-thematic words) ANOVA, the last factor being within-participants. We again found only a main 
TABLE 2. Proportions of Different Types of Gist Memories by List

\begin{tabular}{|c|c|c|c|c|}
\hline \multirow[b]{2}{*}{ List Items } & \multicolumn{4}{|c|}{ Lists } \\
\hline & Social Positive & Social Negative & $\begin{array}{r}\text { Intellectual } \\
\text { Positive }\end{array}$ & $\begin{array}{r}\text { Intellectual } \\
\text { Negative }\end{array}$ \\
\hline \multicolumn{5}{|l|}{ Impression Formation } \\
\hline Trait Items & .97 & .93 & .86 & .89 \\
\hline A-thematic Items & .78 & .82 & .84 & .80 \\
\hline \multicolumn{5}{|l|}{ Gist Memories } \\
\hline Social Positive & .81 & .13 & .21 & .18 \\
\hline Social Negative & .12 & .73 & .11 & .20 \\
\hline Intellectual Positive & .65 & .27 & .68 & .17 \\
\hline Intellectual Negative & .12 & .44 & .12 & .61 \\
\hline A-thematic Associates & .20 & .21 & .14 & .20 \\
\hline \multicolumn{5}{|l|}{ Memory } \\
\hline Trait Items & .93 & .93 & .88 & .82 \\
\hline A-thematic Items & .81 & .75 & .83 & .82 \\
\hline \multicolumn{5}{|l|}{ Gist Memories } \\
\hline Social Positive & .59 & .12 & .21 & .09 \\
\hline Social Negative & .15 & .73 & .16 & .18 \\
\hline Intellectual Positive & .48 & .14 & .53 & .11 \\
\hline Intellectual Negative & .14 & .42 & .23 & .54 \\
\hline A-thematic Associates & .47 & .43 & .54 & .36 \\
\hline
\end{tabular}

Note. Proportions of gist memories that corresponded to the same cluster of the Implicit Theory of Personality of the items of the presented list are in bold. $N=149$

effect for Type of Hits, $F(1,141)=18.51, p=.0001, M S e=.03, \eta^{2}=.11$, showing that participants accepted more presented traits than a-thematic words $(M=$ .93 vs. $M=.85$ ). Again, our critical data regard the proportion of "gist memories," ${ }^{2}$ that is, accepted non-presented items that semantically correspond to the cluster of the presented list (see Table 2). We predicted that the difference in false memories between impression formation and memory found under standard recognition test would persist in the gist test. To test this prediction we recoded our gist memories as we did for the standard recognition data into list-matching (proportions of gist memories that correspond to the same trait cluster of the items of the presented list), list mismatching (proportions of gist memories that correspond to a different trait cluster) and A-thematic Associates (non-presented items that are associated with the a-thematic presented words). We performed a 2 Processing Goal (Impression Formation vs. Memory) X 4 Lists (Social Positive vs. Social Negative vs. Intellectual Positive vs. Intellectual Negative) X 3 Type of Gist Memories (List Matching vs. List

2. Technically, in the case of a gist recognition test, the acceptance of a related but non-presented item cannot be considered a false memory because test instruction requested participants to accept precisely this type of item. Thus we will call gist memories to accepted non-presented items that relate to presented stimuli (these items correspond, of course, to false memories in standard recognition tests). 
Mismatching vs. A-thematic Associates) mixed-model ANOVA, the last factor being within-participants. The pattern of results paralleled what we found with the standard test. We obtained a significant Type of Gist Memories main effect. This Type of Gist Memories effect, $F(2,282)=167.95, p=.0001, M S e=$ $.05, \eta^{2}=.50$, revealed that for gist memories, List Matching $(M=.65)$ were much more likely than List Mismatching $(M=.22)$ or A-thematic Associates $(M=.27)$. In addition, we also obtained the critical Processing Goal X Type of Gist Memories interaction, $F(2,282)=15.77, p=.0001, M S e=.05, \eta^{2}=.05$. This interaction shows that whereas the level of false memories was much higher for Impression Formation relative to Memory participants in the case of List Matching $(M=.71$ vs. $M=.60), t(141)=1.99, p=.0484)$, this difference disappeared for List Mismatching $(M=.22$ vs. $M=.20, t<1)$ and reversed for A-thematic Associated gist memories $(M=.18$ vs. $M=.35, t(141)=3.56), p=$ $.0001 .^{3}$ Recall that we include a gist recognition test because the comparison between the data patterns for both types of tests has been used to differentiate between monitoring-based and relational-deficit-based accounts of decreased false memories. The parallel data patterns obtained suggest that the differences between impression formation and memory participants were not due to differences in monitoring efficacy but instead stem from divergent relational deficits. Memory participants (relatively to their Impression Formation counterparts) seem to exhibit an implicit theory of personality relational deficit, in the sense that they committed fewer false memories in standard recognition tests and accepted fewer gist items in the gist test for items that corresponded to the same cluster of the items of the presented list items. Conversely, Impression Formation relative to Memory participants display a semantic relational deficit, in the sense that they committed fewer false memories in standard recognition tests and accepted fewer gist items in the gist test for non-presented associates of presented a-thematic items. We will explore the consequences of these results in the final section.

\section{GENERAL DISCUSSION}

The reported results support our claim that false memories can stem from the implicit theory of personality semantic structure as participants form impressions of personality. More specifically, the level of false memories obtained under impression formation instructions was higher than the level obtained under memory instructions.

Moreover, the inclusion of a-thematic words among personality traits in the study lists led to more false alarms under memory instructions than under impression formation both in the standard and the gist recognition test. Thus, our results point to the critical importance of impression formation goals in the activation of a specialized semantic structure. From these results, we suggest that different encoding goals can lead to the activation of somewhat different

3. A complex Processing Goal X List Valence X Type of Gist Memory was also significant suggesting that the above reported two-way interaction was stronger for positive than for negative lists. 
semantic structures. Once these specialized associative structures are active they seem even to interfere with the activation of the default association of the available stimuli, as it was the case with our a-thematic words. Under impression formation goals, the default semantic associates of the a-thematic words were much less often accepted in the gist recognition test than under a memory goal. Although we believe that the comparison between results from the standard and the gist recognition tests is suggestive, we must acknowledge that because the standard recognition test always preceded the gist recognition test, performance in the former may have influenced performance in the latter. Thus future research should examine this possibility by manipulating the nature of test factor in a between-participants design.

Taken together, these results suggest that the Implicit Theory of Personality may function as a specialized semantic structure that is activated when the goal of impression formation is operative. This suggestion converges with recent work that advocates that social cognition may involve more than the mere recruitment of basic generic cognitive processes-it may involve the participation of specialized brain structures and dedicated cognitive processes that are activated whenever a social cognitive goal (e.g., impression formation) is pursued (Mitchell, Macrae, \& Banaji, 2004). Moreover, although there have been previous attempts to characterize the cognitive nature of the Implicit Theory of Personality (Cantor \& Mischel, 1977, 1979; Schneider \& Blankemeyer, 1983), we believe that both the present characterization of the Implicit Theory of Personality as a conditional associative structure and its exploration in an experimental paradigm that allows for systematic comparison with other associative structures (the DRM) represent a step forward to our understanding of the processes underlying impression formation.

In addition, the conditional nature of the Implicit Theory of Personality associative structure may contribute to better understand false memories and associative memory in general. For instance, it seems that whereas the standard DRM paradigm makes use of unconditional or, at least, default associative structures, equivalent effects have been shown to occur only when the stimuli are studied under appropriate instructions (for instance, the false memories of numeric associates only occur if participants are carrying the appropriate arithmetic operations at encoding, see Pesta et al., 2001). In sum, the systematic comparison of the characteristics of what we here labeled conditional and unconditional associative structures is an important endeavor that has been largely ignored. We hope to have contributed with a first step to change this state of affairs.

Finally, we deem our success in adapting the DRM paradigm to impression formation as particularly noteworthy because we selected trait-words not from a list of associates but from casual descriptions of real people. Although theoretically relevant false memories obtained using the DRM paradigm could be a result of using particular lists of stimuli that participants would not previously have learned to monitor adequately; in our case, we started with trait lists based on dimensions that underlie common descriptions of people, asked par- 
ticipants to form impressions of hypothetical targets described by such traits, and obtained levels of false memories of considerable magnitude. Hence, our successful adaptation of the DRM paradigm to impression formation supports the notion that false memories are not the outcome of a clever but unusual study list but instead the cost of a flexible associative structure.

\section{REFERENCES}

Asch, S. E. (1946). Forming impressions of personality. Journal of Abnormal and Social Psychology, 41, 258-290.

Berman, J. B., \& Kenny, D. A. (1976). Correlational bias in observer ratings. Journal of Personality and Social Psychology, 34, 263-273.

Brainerd, C. J., Wright, R., Reyna, V. F., \& Payne, D. G. (2002). Dual-retrieval processes in recall. Journal of Memory and Language, 46, 120-152.

Brown, R. (1986). Social psychology, 2nd ed. New York: Free Press.

Bruner, J. S., Shapiro, D., \& Tagiuri, R. (1958). The meaning of traits in isolation and combination. In R. Tagiuri \& L. Petrullo (Eds.), Person perception and interpersonal behavior (pp. 277-288). Stanford, CA: Stanford University Press.

Bruner, J. S., \& Tagiuri, R. (1954). The perception of people. In G. Lindzey (Ed.), Handbook of social psychology (Vol. 2, pp. 634-654). Reading, MA: AddisonWesley.

Cantor, N., \& Mischel, W. (1977). Prototypes in person perception. In L. Berkowitz (Ed.), Advances in experimental social psychology (Vol. 12, pp. 3-52). New York: Academic.

Cantor, N., \& Mischel, W. (1979). Prototypicality and personality: Effects on free recall and personality impressions. Journal of Research in Personality, 13, 187-205.

Chartrand, T. L., \& Bargh, J. A. (1996). Automatic activation of impression formation and memorization goals: Nonconscious goal priming reproduces effects of explicit task instructions.
Journal of Personality and Social Psycho$\log y, 71,464-478$.

Deese, J. (1959). On the prediction of occurrence of particular verbal intrusions in immediate recall. Journal of Experimental Psychology, 58, 17-22.

Fiske, S. T., Cuddy, A. J. C., \& Glick, P. (2007). Universal dimensions of social perception: Warmth and competence. Trends in Cognitive Science, 11, 77-83.

Hamilton, D. L., Katz, L. B., \& Leirer, V. O. (1980). Cognitive representation of personality impressions: Organizational processes in first impression formation. Journal of Personality $\mathcal{E}$ Social Psychology, 39, 1050-1063.

Hege, A. C. G., \& Dodson, C. S. (2004). Why distinctive information reduces false memories: Evidence for both impoverished relational-encoding and distinctiveness heuristic accounts. Journal of Experimental Psychology: Learning, Memory, and Cognition, 30, 787-795.

Lichtenstein, M., \& Srull, T. K. (1987). Processing objectives as a determinant of the relationship between recall and judgment. Journal of Experimental Social Psychology, 23, 93-118.

Mitchell, J. P., Macrae, C. N., \& Banaji, M. R. (2004). Encoding-specific effects of social cognition on the neural correlates of subsequent memory. Journal of $\mathrm{Neu}$ roscience, 24, 4912-4917.

Passini, F. T., \& Norman, W. T. (1966). A universal conception of personality structure? Journal of Personality and Social Psychology, 4, 44-49.

Payne, D. G., Elie, C. J., Blackwell, J. M., \& Neuschatz, J. S. (1996). Memory illusions: Recalling, recognizing, and rec- 
ollecting events that never occurred. Journal of Memory and Language, 35, 261-285.

Pesta, B., Sanders, R., \& Murphy, M. (2001). Misguided multiplication: Creating false memories with numbers rather than words. Memory $\mathcal{E}$ Cognition, 29, 478-483.

Roediger, H. L., III. (1996). Memory illusions. Journal of Memory and Language, 35, 76-100.

Roediger, H. L., III, \& McDermott, K. B. (1995). Creating false memories: Remembering words not presented in lists. Journal of Experimental Psychology: Learning, Memory, and Cognition, 21, 803-814.

Roediger, H. L., III, Watson, J. M., McDermott, K. B., \& Gallo, D. A. (2001). Factors that determine false recall: A multiple regression analysis. Psychonomic Bulletin \& Review, 8, 385-407.

Rosenberg, S., Nelson, C., \& Vivekanathan, P. S. (1968). A multidimensional approach to the structure of personality impressions. Journal of Personality and Social Psychology, 9, 283-294.

Rosenberg, S., \& Sedlak, A. (1972). Structural representations of implicit personality theory. In L. Berkowitz (Ed.), Advances in experimental social psychology (Vol. 6, pp. 235-297). New York: Academic.

Schacter, D. L., Cendan, D. L., Dodson, C. S., \& Clifford, E. R. (2001). Retrieval conditions and false recognition: Testing the distinctiveness heuristic. Psychonomic Bulletin and Review, 8, 827-833.

Schneider, D. J., \& Blankmeyer, B. L. (1983). Prototype salience and implicit personality theories. Journal of Personality and Social Psychology, 44, 712-722.

Sommers, M. S., \& Lewis, B. P. (1999). Who really lives next door: Creating false memories with phonological neighbors. Journal of Memory and Language, 40, 83-108.

Thorndike, E. L. (1920). A constant error in psychological ratings. Journal of Applied Psychology, 4, 469-477. 